\title{
Microwave Spectroscopy Reveals the Quantum Geometric Tensor of Topological Josephson Matter
}

\author{
R. L. Klees $\odot,{ }^{1}$ G. Rastelliø, ${ }^{1,2}$ J. C. Cuevas $\oplus^{3}{ }^{3}$ and W. Belzig ${ }^{1}$ \\ ${ }^{1}$ Fachbereich Physik, Universität Konstanz, D-78457 Konstanz, Germany \\ ${ }^{2}$ Zukunftskolleg, Universität Konstanz, D-78457 Konstanz, Germany \\ ${ }^{3}$ Departamento de Física Teórica de la Materia Condensada and Condensed Matter Physics Center (IFIMAC), \\ Universidad Autónoma de Madrid, E-28049 Madrid, Spain
}

(Received 28 June 2019; revised manuscript received 13 November 2019; accepted 6 April 2020; published 13 May 2020)

\begin{abstract}
Quantization effects due to topological invariants such as Chern numbers have become very relevant in many systems, yet key quantities such as the quantum geometric tensor providing local information about quantum states remain experimentally difficult to access. Recently, it has been shown that multiterminal Josephson junctions constitute an ideal platform to synthesize topological systems in a controlled manner. We theoretically study properties of Andreev states in topological Josephson matter and demonstrate that the quantum geometric tensor of Andreev states can be extracted by synthetically polarized microwaves. The oscillator strength of the absorption rates provides direct evidence of topological quantum properties of the Andreev states.
\end{abstract}

DOI: 10.1103/PhysRevLett.124.197002

Introduction.-Presently, there is huge interest in condensed matter physics in topologically nontrivial systems and, in the last two decades, there has been great effort to find novel types of topological quantum matter such as topological insulators [1,2], topological semimetals [3], or topological superconductors [4]. The topological phase is often related to isolated singularities in the band structure at which two energy bands intersect $[5,6]$. In the case of topological superconductors, Bogoliubov quasiparticles at zero energy, called Majorana zero modes, could potentially be used in topologically protected quantum computation [4]. The existence of zero-energy modes in such systems is topologically protected [7], which recently has been confirmed in experiments on superconducting three-terminal junctions [8]. Actually, Andreev bound states (ABS) in superconducting weak links, also known as Josephson junctions, have also been proposed for implementing qubits $[9,10]$. ABS can be easily tuned if the junctions are embedded in an rf superconducting quantum interference device (SQUID) and can be experimentally accessed and coherently manipulated by microwave [11-14], tunneling [15], and supercurrent spectroscopy [16].

Recently, multiterminal Josephson junctions (MJJs) made of conventional superconductors have been predicted to exhibit nontrivial topology for four [17-22] and three [23-27] leads. In such systems there is no need for exotic topological materials, although multiterminal topological nanowires have been discussed as well [27]. In MJJs the quantized transconductance across two terminals is a manifestation of the integer-valued Chern number $[17,20,21,27]$. Alternatively, Floquet states in periodically driven Josephson systems with connectivity simpler than
MJJs can also show nontrivial topology [28,29]. Although it is challenging to fabricate MJJs [30], a realization of a three-terminal superconducting junction in a doubleSQUID configuration and the investigation of its topological properties has already been reported [31]. First experiments towards ballistic MJJs have been performed, too $[32,33]$.

Since the Chern number follows from integrating the Berry curvature over periodic parameters, accessing the more fundamental local properties contained in the quantum geometric tensor (QGT), i.e., the Fubini-Study metric tensor and the Berry curvature, provides additional information about the geometry of the state space manifolds [34]. There have been several proposals how to measure the elements of the QGT, e.g., via the noise spectral functions [35] proposed also for electronic solid state systems [36] or via nonadiabatic periodic modulation of the space-defining parameters [37]. In fact, local topological properties can also be revealed by the quantized spectroscopic response under (nonadiabatic) circular drive [38,39], which has already been successfully carried out in Floquet states of ultracold fermionic atoms under time-dependent drive [40,41]. Similarly, a nontrivial (Floquet) topology was achieved in superconducting qubits $[42,43]$ generated by custom-built engineered time-dependent drives.

In this Letter, we present a straightforward way to experimentally access the full QGT in MJJs. We show how to extract the elements of the QGT of the ground state manifold of the low-energy ABS hosted in MJJs by measuring the absorption rates under a weak timedependent perturbation. Such linear response measurements have now become standard in ABS spectroscopy [11-13]. 

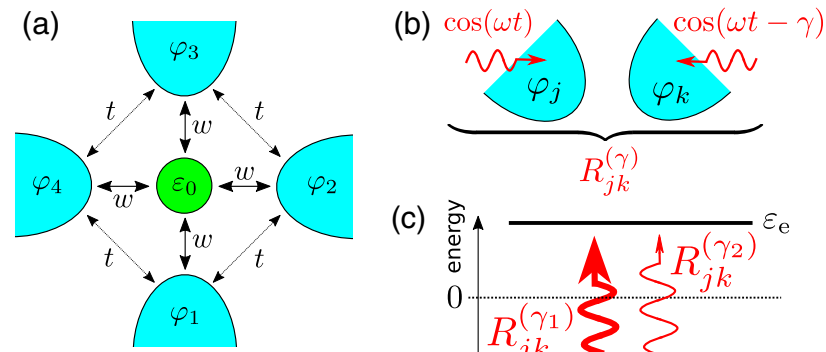

(c)

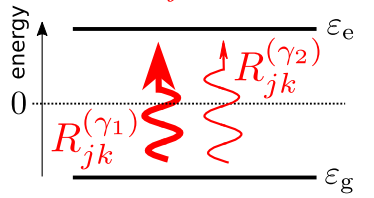

(d)

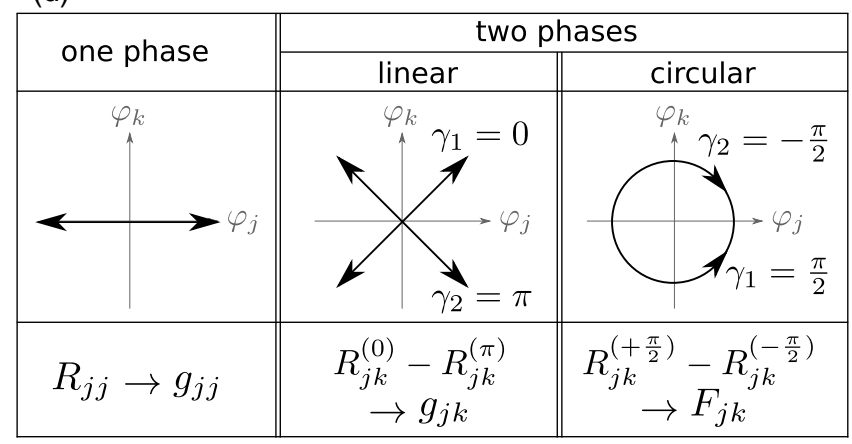

FIG. 1. Application of polarized microwave spectroscopy in MJJs. (a) Microscopic model of the four terminals. Four superconducting leads, each with a phase $\varphi_{j}(j=1,2,3,4)$, are connected to a normal quantum dot with level $\varepsilon_{0}$ via the couplings $w$. The nearest leads are also directly connected to each other by the couplings $t \ll w$. (b) A periodic modulation of two phases $\varphi_{j}$ and $\varphi_{k}(j \neq k)$ at frequency $\omega$ leads to transitions with rates of absorption $R_{j k}^{(\gamma)}$, where $\gamma$ is the relative phase between the two modulations. (c) Two measurements with different relative phases $\gamma_{1} \neq \gamma_{2}$ lead to different transition rates $R_{j k}^{\left(\gamma_{1}\right)} \neq R_{j k}^{\left(\gamma_{2}\right)}$ between the ground state at energy $\varepsilon_{g}$ and the excited state at energy $\varepsilon_{e}$. (d) Schema of how to extract the elements of the quantum geometric tensor $\chi_{j k}=g_{j k}-i F_{j k} / 2$, where $g_{j k}$ is the metric tensor and $F_{j k}$ is the Berry curvature. Driving of one phase $\varphi_{j}$ allows for the detection of the diagonal elements $g_{j j}$, while linear (circular) driving of two phases $\varphi_{j}$ and $\varphi_{k}(j \neq k)$ allows for the extraction of the off-diagonal elements $g_{j k}\left(F_{j k}\right)$.

To this end, we conceive a concrete and feasible experimental way to implement synthetically linear or circular polarized microwave absorption spectroscopy in MJJs. Figure 1 presents the specific example of a four-terminal Josephson junction and summarizes the general protocol to extract the full information of the metric tensor and the Berry curvature via microwave absorption spectroscopy. The latter represents our main result. Our proposed method can be used in a large variety of topological Josephson matter and, therefore, provides an unprecedented insight into the nature of quantum states in these systems. Finally, by integrating the absorption rate differences related to the Berry curvature, our approach allows us to measure the Chern number in MJJs complementary to transconductance measurements.
Model and effective Hamiltonian.-For the sake of concreteness, we consider a four-terminal setup sketched in Fig. 1(a) consisting of four superconducting (SC) terminals connected to a normal conducting region that consists of a single level, noninteracting quantum dot providing a spin-degenerate energy level $\varepsilon_{0}$. We assume that the SC leads are described by standard BCS-type mean-field Hamiltonians, with a pairing potential $\Delta>0$ and phase $\varphi_{j} \in[0,2 \pi)$ for $j=1, \ldots, 4$, and that the quantum dot is coupled to the leads with tunneling coupling strength $w>0$, while the leads are coupled to each other with a tunneling coupling strength $t>0$.

In the large-gap limit $\Delta \rightarrow \infty$ and for $t / w \ll 1$, the effective Hamiltonian describing the pair of ABS on the dot reads $H=\Psi^{\dagger} H_{0} \Psi$, where $\Psi^{\dagger}=\left(d_{\uparrow}^{\dagger}, d_{\downarrow}\right)$ is the Nambu spinor consisting of an electronic annihilation (creation) $d_{\sigma}^{(\dagger)}$ operator of spin $\sigma$ on the dot and $H_{0}=\boldsymbol{d} \cdot \boldsymbol{\tau}$ describes a pseudospin $\boldsymbol{\tau}=\left(\tau_{1}, \tau_{2}, \tau_{3}\right)^{\mathrm{T}}$ (Pauli matrices in Nambu space) in an effective magnetic field

$$
\boldsymbol{d}=\left(\begin{array}{c}
\Gamma \sum_{j=1}^{4} \cos \varphi_{j} \\
-\Gamma \sum_{j=1}^{4} \sin \varphi_{j} \\
\varepsilon_{0}-2 t_{0} \Gamma \sum_{j=1}^{4} \cos \left(\varphi_{j}-\varphi_{j+1}\right)
\end{array}\right)
$$

controlled by the SC phases $\varphi_{j}$ [44]. Here, $\Gamma=\pi N_{0} w^{2}$ and $t_{0}=\pi N_{0} t$, where $N_{0}$ is the normal density of states in the leads at the Fermi level.

Andreev states and topology.-The low-energy Hamiltonian $H_{0}$ defines a two-level system with a ground state (GS) $|g\rangle$ and an excited state $|e\rangle$, where $H_{0}|e / g\rangle=\varepsilon_{e / g}|e / g\rangle$. The pair of ABS has energies given by $\varepsilon_{e / g}= \pm d$, where we define $d=|\boldsymbol{d}|$. In the fourterminal case only three SC phases are independent and, therefore, gauge invariance allows us to set one SC phase to zero (from now on we set $\varphi_{4}=0$ ). The remaining three SC phases $\boldsymbol{\varphi}=\left(\varphi_{1}, \varphi_{2}, \varphi_{3}\right) \in[0,2 \pi)^{3}$ define a first Brillouin zone (FBZ) in analogy to the quasimomentum space of a periodic crystal. The spectrum $\varepsilon_{e / g}$ is shown in Figs. 2(a)-2(d) for several values of $\varphi_{3}$ which show zero-energy Weyl nodes $\boldsymbol{\varphi}_{W}$ separating different gapped phases. From the constraint $d\left(\boldsymbol{\varphi}_{W}\right)=0$, we find that Weyl nodes only appear if $-8 \leq m \leq 0$ with $m=\varepsilon_{0} / t_{0} \Gamma$. There are four Weyl nodes $\boldsymbol{\varphi}_{W}^{(s)}=\left(\varphi_{W, 1}^{(s)}, \varphi_{W, 2}^{(s)}, \varphi_{W, 3}^{(s)}\right), s=1,2,3$, 4 , located at (modulo $2 \pi$ in each direction)

$$
\begin{gathered}
\boldsymbol{\varphi}_{W}^{(1)}=(-\delta, \pi-\delta, \pi), \\
\boldsymbol{\varphi}_{W}^{(2)}=(\delta, \delta-\pi, \pi), \\
\boldsymbol{\varphi}_{W}^{(3)}=(\pi, \pi-\delta,-\delta), \\
\boldsymbol{\varphi}_{W}^{(4)}=(\pi, \delta-\pi, \delta),
\end{gathered}
$$



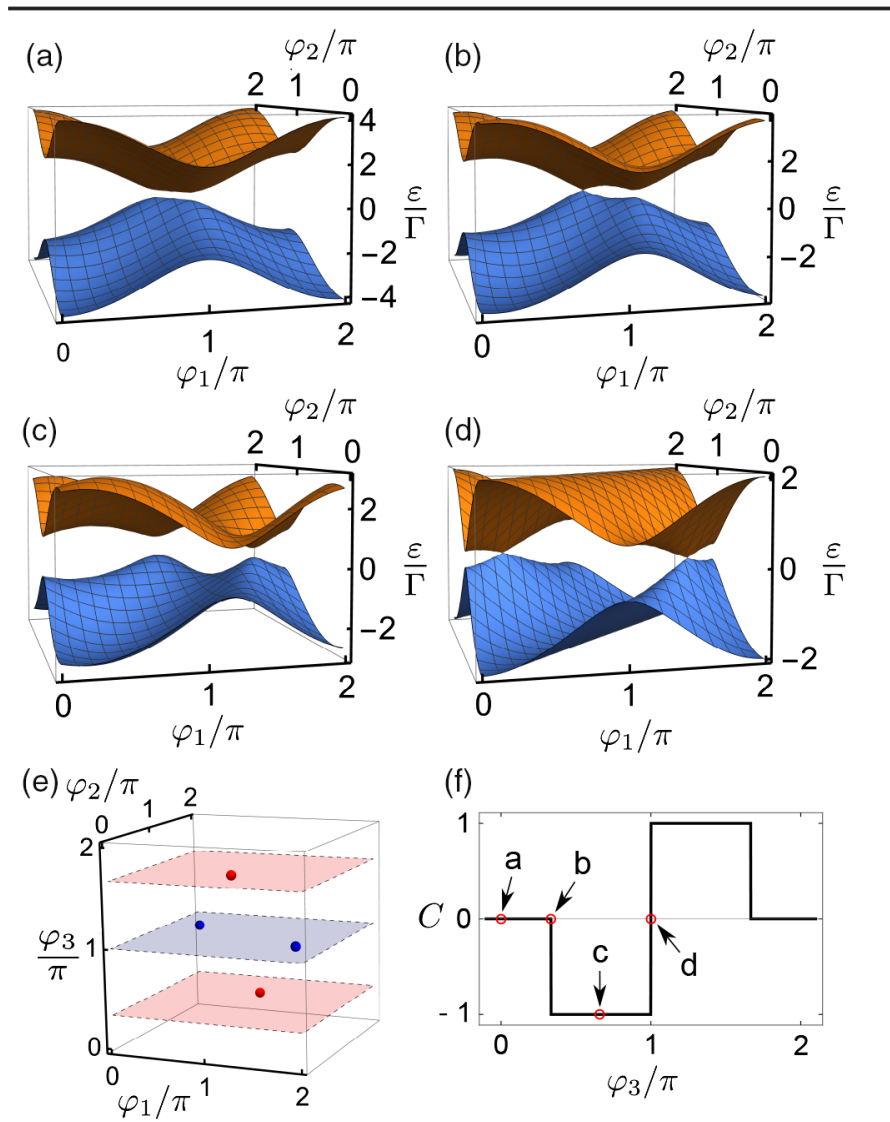

FIG. 2. Band structure, Weyl nodes, and Chern number in the four-terminal junction. (a)-(d) Energy spectrum $\varepsilon_{e / g}= \pm d$ for (a) $\varphi_{3}=0$, (b) $\varphi_{3}=\pi / 3$, (c) $\varphi_{3}=2 \pi / 3$, (d) $\varphi_{3}=\pi$, respectively. (e) Locations of the four Weyl nodes in the FBZ. Blue (red) Weyl nodes carry a topological charge $c=+1(c=-1)$, see Ref. [44] for details. The Chern number becomes nontrivial only if the $\left(\varphi_{1}, \varphi_{2}\right)$ plane of integration lies between two opposing charges. (f) Chern number $C$ as a function of $\varphi_{3}$. The points a, b, $\mathrm{c}$ and $\mathrm{d}$ correspond to the values of $\varphi_{3}$ in panels (a),(b),(c) and (d), respectively. Common parameters for all panels: $t_{0}=0.1, \varepsilon_{0} / \Gamma=-0.2$.

where $\delta=\arccos (1+m / 4)$, each of them carrying a topologically positive or negative charge $[44,45]$. The locations of these zero-energy bound states in the FBZ are shown in Fig. 2(e). The existence of well-separated Weyl nodes is robust against small variations of the coupling constants. These variations simply move the Weyl points in parameter space away from the locations given in Eq. (2) leaving the topological structure of the system intact. Only if the Hamiltonian drastically differs from the presented one, Weyl nodes might merge and annihilate. This happens, for instance, if we add a nextnearest-neighbor coupling between leads $1-3$ and 2-4 of the same magnitude as the nearest-neighbor couplings. We also remark that the existence of zero-energy solutions $\boldsymbol{\varphi}_{W}^{(s)}$ is crucially linked to the presence of a hopping $(t \neq 0)$ directly connecting nearest leads. The latter allows for different interfering paths for particles between every two neighboring leads. In the absence of these paths (i.e., $t=0$ ), the gap between the ABS cannot be closed for any $\varepsilon_{0} \neq 0$ and the system stays topologically trivial.

All SC phases play the role of synthetic $U(1)$ gauge fields for which we define a gauge connection 1-form $\mathcal{A}=$ $\sum_{j} A_{j} d \varphi_{j}$ of the GS $|g\rangle$ [46], where $A_{j}=i\left\langle g \mid \partial_{j} g\right\rangle$ is the Berry connection [5] and $\partial_{j} \equiv \partial / \partial \varphi_{j}$. The Chern number of the GS manifold is encoded in the gauge-invariant curvature 2-form $\mathcal{F}=d \mathcal{A}=(1 / 2) \sum_{j k} F_{j k} d \varphi_{j} \wedge d \varphi_{k}$, where $F_{j k}=\partial_{j} A_{k}-\partial_{k} A_{j}$ is the Berry curvature [47]. For our particular two-level Hamiltonian $H_{0}$, the Berry curvature of the GS in the gapped phase $(d>0)$ can be expressed as $F_{j k}(\boldsymbol{\varphi})=\boldsymbol{n} \cdot\left[\left(\partial_{j} \boldsymbol{n}\right) \times\left(\partial_{k} \boldsymbol{n}\right)\right] / 2[2,44]$ via the normalized effective magnetic field $\boldsymbol{n}=\boldsymbol{d} / d$. Defining a Chern number for fixed $\varphi_{3}$ via

$$
C\left(\varphi_{3}\right)=\frac{1}{2 \pi} \int_{0}^{2 \pi} d \varphi_{1} \int_{0}^{2 \pi} d \varphi_{2} F_{12}(\boldsymbol{\varphi}),
$$

we observe topologically nontrivial regions with nonzero Chern number for certain values of $\varphi_{3}$ [Fig. 2(f)]. Depending on the topological charge of a Weyl node, the Chern number changes by \pm 1 for each Weyl node that is crossed while moving the $\left(\varphi_{1}, \varphi_{2}\right)$ plane of integration along the $\varphi_{3}$ axis. Therefore, the finite jumps of $C$ are associated with the values $\varphi_{W, 3}=\pi$ and $\varphi_{W, 3}= \pm \delta$. In the shown case for $m=-2$ in Figs. 2(e) and 2(f), the three values of a topological phase transition are $\varphi_{W, 3}=$ $\pi / 3, \pi, 5 \pi / 3$ in the FBZ.

Microwave spectroscopy of quantum geometry.-The gauge-invariant hermitian QGT of the GS is defined as [34]

$$
\chi_{j k}=\left\langle\partial_{j} g|(1-|g\rangle\langle g|)| \partial_{k} g\right\rangle .
$$

The QGT contains the symmetric (Fubini-Study) metric tensor $g_{j k}=\operatorname{Re}\left(\chi_{j k}\right)$ measuring the "distance" between two adiabatically connected states and the antisymmetric Berry curvature $F_{j k}=-2 \operatorname{Im}\left(\chi_{j k}\right)$ containing information about the geometrical phase acquired during an adiabatic change of parameters. Similar to the Berry curvature, also the metric tensor $g_{j k}$ can be conveniently calculated from the normalized effective magnetic field $\boldsymbol{n}$ via $g_{j k}=\left(\partial_{j} \boldsymbol{n}\right)$. $\left(\partial_{k} \boldsymbol{n}\right) / 4[44,48]$.

Let us first show how the diagonal components of the QGT, $\chi_{j j}=g_{j j}$, can be obtained. For this purpose, we modulate one of the SC phases according to $\varphi_{j} \rightarrow \varphi_{j}+(2 A / \hbar \omega) \cos (\omega t)$, with a frequency $\omega$ and for $(A / \hbar \omega) \ll 1$ [see Fig. 1(b)], where $A$ is a coupling parameter, $\hbar$ is Planck's constant, and $t$ is time. The resulting Hamiltonian to linear order becomes $H=H_{0}+$ $2 A\left(\partial_{j} H_{0}\right) \cos (\omega t) / \hbar \omega$ giving rise to transitions between the two states with absorption rates $R_{j j}=r_{j j} \delta(2 d-\hbar \omega)$ by applying Fermi's golden rule [see Fig. 1(c)]. The oscillator strength is then given by $[37,44]$ 


$$
r_{j j}=\frac{2 \pi}{\hbar} A^{2} g_{j j}
$$

The oscillator strength, or the line intensity, can be obtained simply by integration over the proper frequency range, around $\hbar \omega \approx \varepsilon_{e}-\varepsilon_{g}=2 d$. Indeed, Eq. (5) is valid even in the presence of a finite broadening of the line, as it is expected in microwave experiments.

Furthermore, the off-diagonal elements are obtained by time-periodic modulation of two phases as shown in Fig. 1(b). Depending on the relative phase difference $\gamma$ between both modulations, one obtains the off-diagonal elements of the QGT $\chi_{j k}=g_{j k}-i F_{j k} / 2$. For $j \neq k$, we use the modulations [see Fig. 1(d)]

$$
\begin{gathered}
\varphi_{j} \rightarrow \varphi_{j}+(2 A / \hbar \omega) \cos (\omega t), \\
\varphi_{k} \rightarrow \varphi_{k}+(2 A / \hbar \omega) \cos (\omega t-\gamma),
\end{gathered}
$$

where we again assume $(A / \hbar \omega) \ll 1$. As before, we obtain the Hamiltonian to linear order

$H=H_{0}+\frac{2 A}{\hbar \omega}\left(\frac{\partial H_{0}}{\partial \varphi_{j}} \cos (\omega t)+\frac{\partial H_{0}}{\partial \varphi_{k}} \cos (\omega t-\gamma)\right)$,

from which we obtain the transition absorption rates $R_{j k}^{(\gamma)}=$ $r_{j k}^{(\gamma)} \delta(2 d-\hbar \omega)$ via Fermi's golden rule. The oscillator strength is given by $[37,44]$

$$
r_{j k}^{(\gamma)}=\frac{2 \pi}{\hbar} A^{2}\left(g_{j j}+g_{k k}+2 g_{j k} \cos \gamma+F_{j k} \sin \gamma\right) .
$$

By performing two subsequent measurements with $\gamma_{1}=0$ and $\gamma_{2}=\pi$ (orthogonal linear polarizations), we can extract the off-diagonal part of the metric tensor $g_{j k}$, while two measurements with $\gamma_{1}=\pi / 2$ and $\gamma_{2}=-\pi / 2$ (right- and left-handed circular polarization) can be used to measure the Berry curvature $F_{j k}$, i.e.,

$$
\begin{array}{r}
r_{j k}^{(0)}-r_{j k}^{(\pi)}=\frac{8 \pi}{\hbar} A^{2} g_{j k}, \\
r_{j k}^{(+\pi / 2)}-r_{j k}^{(-\pi / 2)}=\frac{4 \pi}{\hbar} A^{2} F_{j k} .
\end{array}
$$

As this gives direct visible evidence about the topological phase of the system, we show the relation between the oscillator strengths for circular drives and the resulting Berry curvatures according to Eq. (9b) for the trivial and the topological phase in Fig. 3 [49].

Finally, let us recall that once the Berry curvature $F_{j k}$ is extracted, the Chern number $C$ automatically follows from an integration of $F_{j k}$ over the corresponding two SC phases $\varphi_{j}$ and $\varphi_{k}$, see Eq. (3).

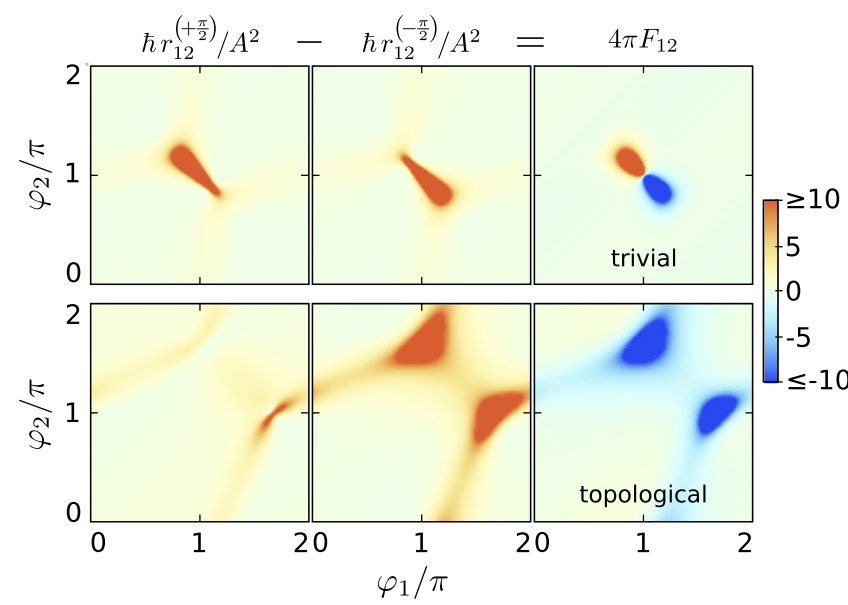

FIG. 3. Oscillator strengths for right- and left-handed circularly polarized absorption (first and second column). The difference shown in the third column is the Berry curvature [Eq. (9b)]. The upper and lower rows correspond to the trivial $\left(\varphi_{3}=0\right)$ and the topological $\left(\varphi_{3}=2 \pi / 3\right)$ phase, respectively [cf. Fig. 2(f)]. The parameters are $t_{0}=0.1, \varepsilon_{0} / \Gamma=-0.2$.

Discussion.-We have presented a protocol to experimentally measure the QGT of topological Josephson matter via generalized microwave spectroscopy in which different forms of synthetic polarizations are applied. The SC phases play the role of quasimomenta in analogy to topological insulators. However, the SC phases can be individually fixed and controlled by SQUID loops, as achieved in the experiments in Refs. [8] and [31]. The modulation of SC phases can be performed by varying the magnetic fluxes in the SQUID loops with a small ac drive, as reported in the spectroscopy experiments $[11-13,16]$.

This procedure is not limited to a four-terminal junction, but can be universally applied to any multiterminal Josephson device. For instance, another possible realization of topological Josephson matter comprises three SC terminals and the normal region is subjected to a perpendicular magnetic field enclosing a magnetic flux [26]. This system also supports Weyl nodes and topologically nontrivial states as long as there is a finite direct coupling between the neighboring leads. The low-energy physics on the dot is again described by an effective Hamiltonian of the form $H_{0}=\boldsymbol{d} \cdot \boldsymbol{\tau}$ [44]. The presented microwave protocol can be applied in the same way as before.

We emphasize that our proposed method is an alternative scheme to detect the topological properties beyond the previously suggested transconductance measurements [17] with the possible advantage that no electronic contacts are needed. A further virtue is that our proposal works at low microwave power such that the linear response regime is applicable. We emphasize that MJJs can be intrinsically topological and, hence, do not require the use of designed (eventually strong) time-dependent drives [28]. Because of 
the universal nature of our proposal, it can be applied to various kinds of topological Josephson matter. As long as two SC phases can be addressed independently, the QGT can be determined by engineering the phase difference as we have described. It is fair, however, to point out that a realization of the exact setup described in this work certainly requires some engineering effort because, in particular, one has to control the coupling between the superconducting leads. Furthermore, the large-gap limit of our model does not consider possible parity jumps due to quasiparticle poisoning [50].

To conclude, it will be interesting to apply our method to proposed topologically protected candidates for quantum information hardware in superconductors, like, e.g., Majorana states [51] or parafermions [52]. Because of the central quantum dot, our model is an ideal platform to address strong Coulomb interaction and study its effects on both quantum geometry and topology beyond the weak perturbative regime which has already been explored [53]. However, this goes beyond the scope of the present work.

R. L. K. thanks Robert Hussein for useful discussions. This work was supported by the Deutsche Forschungsgemeinschaft through SFB 767 and Grant No. RA 2810/1. J.C.C. acknowledges the support via the Mercator Program of the DFG in the frame of the SFB 767.

Note added in the proof.-An experimental measurement of the quantum geometric tensor in qubits formed by NV centers in diamond was recently reported in Ref. [54].

[1] C. L. Kane and E. J. Mele, $Z_{2}$ Topological Order and the Quantum Spin Hall Effect, Phys. Rev. Lett. 95, 146802 (2005).

[2] M. Z. Hasan and C.L. Kane, Colloquium: Topological insulators, Rev. Mod. Phys. 82, 3045 (2010).

[3] N. P. Armitage, E. J. Mele, and A. Vishwanath, Weyl and Dirac semimetals in three-dimensional solids, Rev. Mod. Phys. 90, 015001 (2018).

[4] M. Sato and Y. Ando, Topological superconductors: A review, Rep. Prog. Phys. 80, 076501 (2017).

[5] M. V. Berry, Quantal phase factors accompanying adiabatic changes, Proc. R. Soc. A 392, 45 (1984).

[6] A. Bansil, H. Lin, and T. Das, Colloquium: Topological band theory, Rev. Mod. Phys. 88, 021004 (2016).

[7] L. Fu and C. L. Kane, Superconducting Proximity Effect and Majorana Fermions at the Surface of a Topological Insulator, Phys. Rev. Lett. 100, 096407 (2008).

[8] G. Yang, Z. Lyu, J. Wang, J. Ying, X. Zhang, J. Shen, G. Liu, J. Fan, Z. Ji, X. Jing, F. Qu, and L. Lu, Protected gap closing in Josephson trijunctions constructed on $\mathrm{Bi}_{2} \mathrm{Te}_{3}$, Phys. Rev. B 100, 180501(R) (2019).

[9] N. M. Chtchelkatchev and Y. V. Nazarov, Andreev Quantum Dots for Spin Manipulation, Phys. Rev. Lett. 90, 226806 (2003).
[10] A. Zazunov, V. S. Shumeiko, E. N. Bratus, J. Lantz, and G. Wendin, Andreev Level Qubit, Phys. Rev. Lett. 90, 087003 (2003).

[11] L. Bretheau, Ç. Ö. Girit, H. Pothier, D. Esteve, and C. Urbina, Exciting Andreev pairs in a superconducting atomic contact, Nature (London) 499, 312 (2013).

[12] C. Janvier, L. Tosi, L. Bretheau, Ç. Ö. Girit, M. Stern, P. Bertet, P. Joyez, D. Vion, D. Esteve, M. F. Goffman, H. Pothier, and C. Urbina, Coherent manipulation of Andreev states in superconducting atomic contacts, Science 349, 1199 (2015).

[13] D. J. van Woerkom, A. Proutski, B. van Heck, D. Bouman, J. I. Väyrynen, L. I. Glazman, P. Krogstrup, J. Nygård, L. P. Kouwenhoven, and A. Geresdi, Microwave spectroscopy of spinful Andreev bound states in ballistic semiconductor Josephson junctions, Nat. Phys. 13, 876 (2017).

[14] M. Hays, G. de Lange, K. Serniak, D. J. van Woerkom, D. Bouman, P. Krogstrup, J. Nygård, A. Geresdi, and M.H. Devoret, Direct Microwave Measurement of Andreev-Bound-State Dynamics in a SemiconductorNanowire Josephson Junction, Phys. Rev. Lett. 121, 047001 (2018).

[15] J.-D. Pillet, C. H. L. Quay, P. Morfin, C. Bena, A. Levy Yeyati, and P. Joyez, Andreev bound states in supercurrentcarrying carbon nanotubes revealed, Nat. Phys. 6, 965 (2010).

[16] L. Bretheau, Ç. Ö. Girit, C. Urbina, D. Esteve, and H. Pothier, Supercurrent Spectroscopy of Andreev States, Phys. Rev. X 3, 041034 (2013).

[17] R.-P. Riwar, M. Houzet, J. S. Meyer, and Y. V. Nazarov, Multi-terminal Josephson junctions as topological matter, Nat. Commun. 7, 11167 (2016).

[18] T. Yokoyama and Y. V. Nazarov, Singularities in the Andreev spectrum of a multiterminal Josephson junction, Phys. Rev. B 92, 155437 (2015).

[19] T. Yokoyama, J. Reutlinger, W. Belzig, and Y. V. Nazarov, Order, disorder, and tunable gaps in the spectrum of Andreev bound states in a multiterminal superconducting device, Phys. Rev. B 95, 045411 (2017).

[20] E. Eriksson, R.-P. Riwar, M. Houzet, J. S. Meyer, and Y. V. Nazarov, Topological transconductance quantization in a four-terminal Josephson junction, Phys. Rev. B 95, 075417 (2017).

[21] H.-Y. Xie, M. G. Vavilov, and A. Levchenko, Weyl nodes in Andreev spectra of multiterminal Josephson junctions: Chern numbers, conductances, and supercurrents, Phys. Rev. B 97, 035443 (2018).

[22] E. V. Repin, Y. Chen, and Y. V. Nazarov, Topological properties of multiterminal superconducting nanostructures: Effect of a continuous spectrum, Phys. Rev. B 99, 165414 (2019).

[23] B. van Heck, S. Mi, and A. R. Akhmerov, Single fermion manipulation via superconducting phase differences in multiterminal Josephson junctions, Phys. Rev. B 90, 155450 (2014).

[24] C. Padurariu, T. Jonckheere, J. Rech, R. Mélin, D. Feinberg, T. Martin, and Y. V. Nazarov, Closing the proximity gap in a metallic Josephson junction between three superconductors, Phys. Rev. B 92, 205409 (2015). 
[25] H.-Y. Xie, M. G. Vavilov, and A. Levchenko, Topological Andreev bands in three-terminal Josephson junctions, Phys. Rev. B 96, 161406 (2017).

[26] J. S. Meyer and M. Houzet, Nontrivial Chern Numbers in Three-Terminal Josephson Junctions, Phys. Rev. Lett. 119, 136807 (2017).

[27] L. P. Gavensky, G. Usaj, and C. A. Balseiro, Topological phase diagram of a three-terminal Josephson junction: From the conventional to the Majorana regime, Phys. Rev. B 100, 014514 (2019).

[28] L. P. Gavensky, G. Usaj, D. Feinberg, and C. A. Balseiro, Berry curvature tomography and realization of topological Haldane model in driven three-terminal Josephson junctions, Phys. Rev. B 97, 220505 (2018).

[29] B. Venitucci, D. Feinberg, R. Mélin, and B. Douçot, Nonadiabatic Josephson current pumping by chiral microwave irradiation, Phys. Rev. B 97, 195423 (2018).

[30] S. R. Plissard, I. van Weperen, D. Car, M. A. Verheijen, G. W. G. Immink, J. Kammhuber, L. J. Cornelissen, D. B. Szombati, A. Geresdi, S. M. Frolov, L. P. Kouwenhoven, and E. P. A. M. Bakkers, Formation and electronic properties of InSb nanocrosses, Nat. Nanotechnol. 8, 859 (2013).

[31] E. Strambini, S. D’Ambrosio, F. Vischi, F. S. Bergeret, Y. V. Nazarov, and F. Giazotto, The $\omega$-SQUIPT as a tool to phaseengineer Josephson topological materials, Nat. Nanotechnol. 11, 1055 (2016).

[32] A. W. Draelos, M.-T. Wei, A. Seredinski, H. Li, Y. Mehta, K. Watanabe, T. Taniguchi, I. V. Borzenets, F. Amet, and G. Finkelstein, Supercurrent flow in multiterminal graphene Josephson junctions, Nano Lett. 19, 1039 (2019).

[33] N. Pankratova, H. Lee, R. Kuzmin, M. Vavilov, K. Wickramasinghe, W. Mayer, J. Yuan, J. Shabani, and V. E. Manucharyan, The multi-terminal Josephson effect, arXiv:1812.06017.

[34] M. Kolodrubetz, D. Sels, P. Mehta, and A. Polkovnikov, Geometry and non-adiabatic response in quantum and classical systems, Phys. Rep. 697, 1 (2017).

[35] M. Kolodrubetz, V. Gritsev, and A. Polkovnikov, Classifying and measuring geometry of a quantum ground state manifold, Phys. Rev. B 88, 064304 (2013).

[36] T. Neupert, C. Chamon, and C. Mudry, Measuring the quantum geometry of Bloch bands with current noise, Phys. Rev. B 87, 245103 (2013).

[37] T. Ozawa and N. Goldman, Extracting the quantum metric tensor through periodic driving, Phys. Rev. B 97, 201117 (2018).

[38] D. T. Tran, A. Dauphin, A. G. Grushin, P. Zoller, and N. Goldman, Probing topology by heating: Quantized circular dichroism in ultracold atoms, Sci. Adv. 3, e1701207 (2017).

[39] D. T. Tran, N. R. Cooper, and N. Goldman, Quantized Rabi oscillations and circular dichroism in quantum Hall systems, Phys. Rev. A 97, 061602 (2018).

[40] N. Fläschner, B. S. Rem, M. Tarnowski, D. Vogel, D.-S. Lühmann, K. Sengstock, and C. Weitenberg, Experimental reconstruction of the Berry curvature in a Floquet Bloch band, Science 352, 1091 (2016).
[41] L. Asteria, D. T. Tran, T. Ozawa, M. Tarnowski, B. S. Rem, N. Fläschner, K. Sengstock, N. Goldman, and C. Weitenberg, Measuring quantized circular dichroism in ultracold topological matter, Nat. Phys. 15, 449 (2019).

[42] X. Tan, D.-W. Zhang, Q. Liu, G. Xue, H.-F. Yu, Y.-Q. Zhu, H. Yan, S.-L. Zhu, and Y. Yu, Topological Maxwell Metal Bands in a Superconducting Qutrit, Phys. Rev. Lett. 120, 130503 (2018).

[43] X. Tan, D.-W. Zhang, Z. Yang, J. Chu, Y.-Q. Zhu, D. Li, X. Yang, S. Song, Z. Han, Z. Li, Y. Dong, H.-F. Yu, H. Yan, S.-L. Zhu, and Y. Yu, Experimental Measurement of the Quantum Metric Tensor and Related Topological Phase Transition with a Superconducting Qubit, Phys. Rev. Lett. 122, 210401 (2019).

[44] See Supplemental Material at http://link.aps.org/ supplemental/10.1103/PhysRevLett.124.197002 for details on the microscopic model of multiterminal Josephson junctions. We also provide detailed information about the calculation of the topological charge of the Weyl points, the derivation of the quantum geometric tensor and its relation to transition rates, and a brief discussion of topology in the three-terminal case.

[45] X. Wan, A. M. Turner, A. Vishwanath, and S. Y. Savrasov, Topological semimetal and Fermi-arc surface states in the electronic structure of pyrochlore iridates, Phys. Rev. B 83, 205101 (2011).

[46] A similar calculation can be done for the excited state $|e\rangle$ which contains the same information since the ABS come in particle-hole symmetric pairs.

[47] M. Nakahara, Geometry, Topology, and Physics (Institute of Physics Publishing, Bristol, 2003).

[48] R. Shankar, Quantum geometry and topology, in Topology and Condensed Matter Physics, edited by S. M. Bhattacharjee, M. Mj, and A. Bandyopadhyay, Texts and Readings in Physical Sciences Vol. 19 (Springer Singapore, Singapore, 2017), pp. 253-279.

[49] A similar figure for the elements of the metric tensor $g$ and its relation to the oscillator strengths for linearly polarized absorptions [Eqs. (5) and (9a)] is shown in Fig. S2, see Ref. [44].

[50] M. Zgirski, L. Bretheau, Q. Le Masne, H. Pothier, D. Esteve, and C. Urbina, Evidence for Long-Lived Quasiparticles Trapped in Superconducting Point Contacts, Phys. Rev. Lett. 106, 257003 (2011).

[51] A. Y. Kitaev, Unpaired Majorana fermions in quantum wires, Phys. Usp. 44, 131 (2001).

[52] J. Klinovaja, A. Yacoby, and D. Loss, Kramers pairs of Majorana fermions and parafermions in fractional topological insulators, Phys. Rev. B 90, 155447 (2014).

[53] P. Marra, A. Braggio, and R. Citro, A zero-dimensional topologically nontrivial state in a superconducting quantum dot, Beilstein J. Nanotechnol. 9, 1705 (2018).

[54] M. Yu, P. Yang, M. Gong, Q. Cao, Q. Lu, H. Liu, S. Zhang, M. B. Plenio, F. Jelezko, T. Ozawa, N. Goldman, S.- L. Zhang, and J.-M. Cai, Experimental measurement of the quantum geometric tensor using coupled qubits in diamond, Nat. Sci. Rev. 7, 254 (2019). 